\title{
Validación convergente de la narrativa prototipo de la depresión en grupos de adultos intermedios y tardíos en Bucaramanga*
}

\section{Convergence validation of the narrative prototypic of the depression in groups of intermediate and delayed adults in Bucaramanga}

\author{
Leonardo Yovany Álvarez Ramírez** \\ Lusdary Flórez Gallo \\ Dalys Mateus \\ Universidad Autónoma \\ de Bucaramanga, Colombia
}

Recibido: 29 de agosto de 2007

Revisado: 30 de octubre de 2007

Aceptado: 25 de noviembre de 2007

\section{Resumen}

El objetivo de esta investigación fue identificar el grado de validación de la narrativa prototipo de la depresión de un grupo de adultos intermedios y tardíos, clasificados como depresivos y otro grupo clasificado como no depresivo de la ciudad de Bucaramanga, diferenciándolos en el grado de su atribución de relación; por medio de una escala de validación convergente de la narrativa prototipo y su patología. Dicha muestra estuvo compuesta por 600 adultos intermedios y tardíos, hombres y mujeres entre los 40 y 75 años de edad, seleccionados de acuerdo con el SCID (Entrevista estructurada de trastornos mentales basada en el DSM-IV), que se utilizó para descartar otras patologías relacionadas con la depresión, y el test de Hamilton (Hamilton Depressión Rating Scale, o HRSD) para identificar el grado de gravedad de la depresión. A su vez, el SCID y el test de Hamilton constituyen los instrumentos de evaluación, así como la narrativa prototipo de la depresión, el instrumento de prueba. Los resultados de los análisis del Chicuadrado y el Gamma indicaron que los sujetos depresivos difieren de los no depresivos, atribuyendo a la narrativa prototipo de la depresión un mayor grado de relación con su vida que los sujetos no depresivos; por lo tanto, a mayor grado de identificación con la narrativa prototipo de la depresión del grupo clasifi-

\footnotetext{
* Este artículo es producto del grupo Cognición en procesos psicosociales y psicoterapéuticos, inscrito en Colciencias y en la dirección de investigaciones de la UNAB.

** Correspondencia: Leonardo Yovany Álvarez Ramírez. Dirección postal: Facultad de Psicología UNAB. Campus El Bosque. Bucaramanga, Colombia. Correos electrónicos: lalvarez4@unab.edu.co; lusda43@hotmail.com; dalemat23@yahoo.com.
} 
cado como depresivo, menor será el grado de identificación con la narrativa prototipo de la muestra clasificada como no depresiva.

Palabras clave: SCID, HRSD, narrativa prototipo de la depresión.

\section{Abstract}

The objective of the present investigation work was to identify the validation degree of the narrative prototype of the depression of a group of depressive intermediate and delayed adults classified as a depressive and another group classified as non depressive of the city of Bucaramanga, differentiating them by the attribution of relation degree through a convergent validation scale from the narrative prototype and its pathology. This sample was composed by 600 intermediate and delayed adults, men and women between the 40 and 75 years old, which were selected according to the SCID (structured interview of mental upheavals based on the DSM-IV), that was used to discard other pathologies related to the depression and, the test of Hamilton (Hamilton Depression Scale Rating, or HRSD) to identify the degree of gravity of the depression, the SCID and the test of Hamilton as well constitute the instruments of evaluation, and the narrative prototype of the depression, the instrument of test. The results of the analyses of the Chi-square, and the Gamma, indicated that the depressive subjects differ from the nondepressive ones, attributing to the narrative prototype of the depression a greater degree of relation with their life that the nondepressive subjects, therefore, to greater degree of identification with the narrative prototype of the depression of the classified group as depressive minor will be the degree of identification with the narrative prototype of the sample classified like nondepressive.

Key words: the SCID, the HRSD and, the narrative prototype of the depression.

Algunos estudios realizados en la investigación de las narrativas prototipo y psicopatología (Gonçalves, Maia, Soares, Duarte, Alves, Henriques \& Pocinho, 1986) han aportado evidencia que respalda la idea de que existen ciertos invariantes prototípicos narrativos en distintas psicopatologías: tóxico-dependientes (Alves, 1993), alcohólicos (Duarte, 1993), anoréxicos (Soares, 1995), agorafóbicos (Henriques, 1995), depresivos (Maia, 1998) y obsesivos-compulsivos (Pocinho, 1999), demostrando además que es posible su identificación en los sujetos que las padecen. Según estos hallazgos, en diferentes entidades psicopatológicas resulta posible, a través de procesos de análisis, la identificación de comunalidades o coincidencias narrativas de diversos pacientes (depresivos, adictos al alcohol, obsesivo-compulsivos, ansiosos) (Gonçalves, Maia, Alves, Soares, Duarte \& Henriques, 1986), quienes validan convergentemente estas narrativas prototipo como representativas de su experiencia patológica (Gonçalves, 2002).
Los estudios de Maia (1998) concluyen que la narrativa prototipo se categorizó desde el reporte que los participantes hacían de su experiencia depresiva en curso. En otro de sus estudios halló que los depresivos diferencian estas narrativas entre otras que no incluyen categorías que les son propias a las que identifican como representativas. Dichas identificaciones se han dado en elementos estructurales que parecen componer una matriz cognitivo-narrativa -contexto, acontecimiento precipitante, respuestas internas, objetivo, acciones, resultado, finalización- (Gonçalves, 1992), que se hallan inmersos en el andamiaje de organización del conocimiento depresivo que hace el individuo en esta condición (Gonçalves, 1995).

El estado preliminar de la investigación acerca del tema ha dejado un campo abierto a la indagación acerca de la posible influencia que pueda llegar a tener algunas variables del sujeto, tales como edad, 
tamaño de la muestra y el contexto sociocultural, entre otras posibles variables correlacionadas.

Por otra parte, la exploración sobre la existencia de un estilo de constricción narrativa psicopatológica, en particular en la depresión, introduce la cuestión problemática acerca de si los adultos mantienen un modo típico de elaboración del relato depresivo como una lectura básica estructural de su experiencia depresiva, ante la cual reaccionan validándola en lo personal.

Emprender un estudio de estas características en adultos de la ciudad de Bucaramanga supone abrir un espacio de cuestionamiento teórico y epistemológico acerca del abordaje del fenómeno psicopatológico y, en particular, de la depresión con profundas implicaciones para la psicoterapia. La exploración de la narrativa prototipo de la depresión en adultos depresivos sirve como una vía de exploración sobre cómo podría ocurrir la construcción del conocimiento relativo a su ser, a su sentir y la manera en la que elaboran una narración acerca del otro y del mundo en sus vidas.

\section{Método}

El diseño de la presente investigación fue de tipo correlacional-transversal. La hipótesis de investigación que se trabajo fue si los adultos intermedios y tardíos depresivos de la ciudad de Bucaramanga difieren de los no depresivos en su atribución de relación de la narrativa prototipo de la depresión con su vida.

\section{Participantes}

La muestra la conformaron 600 adultos intermedios y tardíos con edades comprendidas entre los 40 y los 75 años, pertenecientes al Centro Vida de Atención al Adulto Mayor, Fundación Albeiro Vargas, Hogar Asilo San Antonio de la ciudad de Bucaramanga; 300 de ellos fueron identificados como sujetos depresivos, según criterios del DSM
IV TR, el SCID (entrevista estructurada de trastornos mentales basada en el DSM IV), el test psicométrico de Hamilton y 300 como sujetos sin diagnóstico de depresión. Se seleccionaron participantes con capacidad lectoescritora básica que permitiera la comprensión de los instrumentos.

\section{Instrumentos}

Se utilizaron dos cuestionarios para el diagnóstico previo de la depresión de cada uno de los participantes a través de:

El SCID-I: Entrevista estructurada de trastornos mentales basada en el DSM IV.

- Fiabilidad: es una entrevista completamente estructurada y exige el juicio del entrevistador; la fiabilidad del SCID-I se basa en el conocimiento que de su aplicación tienen los aplicadores.

- Validez: se aprecia en el uso de diagnósticos clínicos convencionales a modo de estándar, puesto que las entrevistas estructuradas han sido especialmente diseñadas para reducir las limitaciones propias de la entrevista clínica no estructurada.

El Test Psicométrico de Depresión de Hamilton: Hamilton Depressión Rating Scale, o HRSD.

Se empleó como instrumento de evaluación de la depresión. Este cuestionario ofrece una medida de la intensidad o gravedad de la depresión. La escala posee consistencia interna (alfa de Cronbach entre 0,76 y 0,92 ). El coeficiente de correlación intraclases es de 0,92. La fiabilidad interobservador oscila -según autores- entre 0,65 y 0,9, 17, 23, 26.

- Validez: su correlación con otros instrumentos de valoración global de la depresión, como la escala de depresión de Montgomery-Asberg, el Inventario de Sintomatología Depresiva y la escala de Melancolía de Beck, oscila entre 0,8 y $0,9,11,23,27$. 


\section{Narrativa prototipo de la depresión}

Escala para la validación convergente de la narrativa prototipo con la experiencia depresiva del sujeto; consiste en dos secciones: la primera, un formato de datos de identificación referidos al género, edad, escolaridad, profesión, tiempo de tratamiento y número de hospitalizaciones, y en la segunda sección, una escala para validar el grado de relación de ésta con la vida del sujeto. La construcción de la narrativa prototipo la llevó a cabo Maia (1998) en Portugal (Europa) para su tesis de maestría titulada Narrativas protótipo e organização do conhecimento na depressão.

\section{Procedimiento}

Los entrevistadores recibieron el entrenamiento en la entrevista SCID. A los participantes los contactaron mediante la presentación de cartas de solicitud de la universidad a través del psicólogo de las instituciones visitadas Centro Vida y Atención al Adulto Mayor, Fundación Albeiro Vargas, Hogar Asilo San Antonio, Hospital San Pablo, y la Unidad Psiquiátrica San Camilo, de la ciudad de Bucaramanga. Participaron de manera voluntaria, previo consentimiento informado.

Se resolvieron las dudas y las inquietudes que los participantes tuvieran acerca de la realización del estudio y de su participación en él, indicando previamente su objetivo, sus características no invasivas y el derecho a suspender o a retirarse del mismo, a conocer los resultados y a no usufructuarse de ellos.

Se hizo una entrevista estructurada SCID a través de la cual se excluyeron otras patologías diferentes de la depresión en los participantes. Posteriormente, se aplicó el test de depresión de Hamilton. Por último, se expuso a cada participante a la narrativa prototipo de la depresión, ante la que debía elegir la opción según el grado

Tabla 1. Comparación de la narrativa prototipo de la depresión de los sujetos según género y edad (adultos intermedios y tardíos)

\begin{tabular}{|c|c|c|c|c|c|c|c|c|c|c|c|}
\hline & \multicolumn{2}{|c|}{1} & \multicolumn{2}{|c|}{2} & \multicolumn{2}{|c|}{3} & \multicolumn{2}{|c|}{4} & \multicolumn{2}{|c|}{5} & \multirow[b]{2}{*}{ TOTAL } \\
\hline EDAD & $F$ & $M$ & $F$ & $M$ & $F$ & $M$ & $F$ & $M$ & $F$ & $M$ & \\
\hline 40 & 2 & 1 & 0 & 0 & 1 & 0 & 0 & 0 & 0 & 0 & 4 \\
\hline 41 & 3 & 1 & 1 & 0 & 2 & 1 & 0 & 1 & 0 & 0 & 9 \\
\hline 42 & 3 & 0 & 0 & 0 & 0 & 0 & 0 & 0 & 0 & 0 & 3 \\
\hline 43 & 1 & 3 & 0 & 1 & 2 & 0 & 0 & 0 & 0 & 0 & 7 \\
\hline 44 & 0 & 4 & 0 & 0 & 0 & 0 & 0 & 1 & 0 & 0 & 5 \\
\hline 45 & 3 & 3 & 0 & 0 & 0 & 0 & 0 & 0 & 1 & 0 & 7 \\
\hline 46 & 2 & 2 & 0 & 0 & 0 & 2 & 1 & 2 & 0 & 1 & 10 \\
\hline 47 & 4 & 2 & 0 & 0 & 2 & 1 & 0 & 1 & 1 & 1 & 12 \\
\hline 48 & 4 & 5 & 0 & 0 & 2 & 2 & 0 & 1 & 1 & 0 & 15 \\
\hline 49 & 8 & 7 & 0 & 0 & 3 & 1 & 1 & 1 & 2 & 1 & 24 \\
\hline 50 & 9 & 8 & 0 & 0 & 6 & 4 & 3 & 1 & 0 & 1 & 32 \\
\hline 51 & 6 & 5 & 1 & 0 & 2 & 1 & 0 & 2 & 1 & 0 & 18 \\
\hline 52 & 7 & 12 & 0 & 0 & 1 & 4 & 1 & 2 & 2 & 0 & 29 \\
\hline 53 & 5 & 12 & 1 & 0 & 1 & 4 & 1 & 1 & 1 & 0 & 26 \\
\hline 54 & 6 & 8 & 1 & 0 & 1 & 6 & 2 & 0 & 0 & 1 & 25 \\
\hline 55 & 9 & 7 & 1 & 1 & 2 & 1 & 1 & 2 & 1 & 0 & 25 \\
\hline 56 & 5 & 2 & 0 & 1 & 7 & 2 & 1 & 1 & 3 & 0 & 22 \\
\hline 57 & 1 & 5 & 1 & 0 & 3 & 2 & 3 & 0 & 1 & 0 & 16 \\
\hline 58 & 4 & 4 & 0 & 0 & 3 & 1 & 2 & 0 & 1 & 1 & 16 \\
\hline 59 & 4 & 2 & 0 & 0 & 2 & 2 & 2 & 1 & 2 & 0 & 15 \\
\hline 60 & 8 & 6 & 0 & 0 & 3 & 3 & 0 & 2 & 4 & 0 & 26 \\
\hline 61 & 5 & 4 & 0 & 0 & 2 & 3 & 0 & 2 & 1 & 2 & 19 \\
\hline 62 & 1 & 4 & 0 & 0 & 2 & 2 & 4 & 1 & 1 & 0 & 15 \\
\hline 63 & 5 & 2 & 1 & 1 & 2 & 4 & 1 & 0 & 0 & 0 & 16 \\
\hline 64 & 4 & 5 & 0 & 0 & 3 & 1 & 2 & 1 & 2 & 1 & 19 \\
\hline 65 & 5 & 4 & 0 & 0 & 5 & 4 & 2 & 4 & 2 & 0 & 26 \\
\hline 66 & 0 & 2 & 1 & 0 & 2 & 2 & 2 & 1 & 1 & 1 & 12 \\
\hline 67 & 7 & 4 & 0 & 0 & 5 & 0 & 2 & 3 & 6 & 1 & 28 \\
\hline 68 & 2 & 1 & 1 & 0 & 3 & 1 & 1 & 1 & 2 & 1 & 13 \\
\hline 69 & 1 & 5 & 0 & 1 & 1 & 1 & 3 & 0 & 2 & 0 & 14 \\
\hline 70 & 4 & 1 & & 0 & 4 & 5 & 1 & 1 & 3 & 4 & 23 \\
\hline 71 & 2 & 5 & 0 & 0 & 2 & 2 & 3 & 1 & 2 & 1 & 18 \\
\hline 72 & 1 & 1 & 0 & 1 & 2 & 3 & 1 & 1 & 2 & 0 & 12 \\
\hline 73 & 1 & 1 & 1 & 2 & 5 & 0 & 2 & 1 & 1 & 0 & 14 \\
\hline 74 & 3 & 0 & 0 & 0 & 0 & 2 & 1 & 0 & 1 & 2 & 9 \\
\hline 75 & 6 & 3 & 0 & 0 & 3 & 2 & 1 & 1 & 0 & 0 & 16 \\
\hline TOTAL & 141 & 141 & 10 & 8 & 84 & 69 & 44 & 37 & 47 & 19 & 600 \\
\hline
\end{tabular}


de identificación, con respecto a su vida, en una escala de 1 a 5 . Donde 5 es total identificación y 1 corresponde a "ninguna relación".

\section{Resultados}

En la tabla 1 se aprecia la distribución por edades y por género de las respuestas a la escala de la narrativa prototipo de la depresión en el grupo estudiado de adultos intermedios y tardíos. Los hallazgos revelan que todas las edades y los dos géneros estuvieron representados en cada una de las escalas, siendo las más calificadas las escalas 1 y 2, correspondientes a la percepción o validación de "ninguna" y "alguna relación con mi vida".

En la tabla 2 se presentan los resultados de la selección previa de sujetos depresivos y no depre- sivos llevada a cabo con la escala de depresión del test de Hamilton. Los datos muestran que los sujetos no deprimidos fueron 300 , los cuales tuvieron puntajes entre 0 y 7 en dicha escala, lo que corresponde a la ausencia de síntomas depresivos. Los sujetos depresivos (300) presentaron puntajes correspondientes a síntomas severos. Esta evaluación coincide con los criterios previos de selección con la entrevista SCID-I, con la cual se evaluó la presencia de depresión en los sujetos de este grupo y la no presencia de la misma en el grupo control.

En la tabla 3 se registra el grado de identificación de la narrativa prototipo de la depresión en los sujetos deprimidos y no deprimidos. En el caso de estos últimos, 282 reportaron no encontrar relación alguna entre su vida y la narrativa prototipo, y 18 de ellos reportaron percibir alguna relación.

Tabla 2. Resumen contingencia de grupo entre narrativa prototipo de la depresión y Hamilton (adultos intermedios y tardíos)

\begin{tabular}{cccc}
\hline $\begin{array}{c}\text { Escala } \\
\text { intensidad }\end{array}$ & $\begin{array}{c}\text { Sujetos } \\
\text { depresivos }\end{array}$ & $\begin{array}{c}\text { Sujetos no } \\
\text { deprimidos }\end{array}$ & Total \\
\hline Sin síntomas depresivos (0-7) & 0 & 300 & 300 \\
Síntomas ligeros (8-10) & 0 & 0 & 0 \\
Síntomas ligeros (10-16) & 0 & 0 & 0 \\
Síntomas severos (18-68 & 300 & 0 & 300 \\
\multicolumn{1}{c}{ Total } & & & 600 \\
\hline
\end{tabular}

Tabla 3. Resumen contingencia de grupo según grado de relación con la narrativa prototipo de la depresión (adultos intermedios y tardíos)

\begin{tabular}{|c|c|c|c|}
\hline Grado relación & Sujeto deprimido & $\begin{array}{l}\text { Sujetos no } \\
\text { deprimidos }\end{array}$ & Total \\
\hline Ninguna relación & 0 & 282 & 282 \\
\hline Alguna relación & 0 & 18 & 18 \\
\hline Tiene relación & 153 & 0 & 153 \\
\hline Mucha relación & 81 & 0 & 81 \\
\hline Total relación & 66 & 0 & 66 \\
\hline Total & 300 & 300 & 600 \\
\hline
\end{tabular}


En el grupo de deprimidos intermedios y tardíos, 153 informaron que la narrativa tenía relación con sus vidas, 81, que la narrativa tenía mucha relación con sus vidas y 66 , que la narrativa tenía total relación con sus vidas.
En la tabla 4 se registran los resultados de la prueba Chi cuadrado, la cual se utilizó para contrastar la hipótesis de investigación, es decir, si los sujetos evaluados depresivos se identificaban con la narrativa prototipo de la depresión y viceversa para el caso de los no depresivos.

Tabla 4. Resultados del cálculo Chi-cuadrado y gamma (adultos intermedios y tardíos)

\begin{tabular}{lc}
\hline Grados de libertad (Gl) & 4 \\
\hline Chi-cuadrado & 10,951 \\
Nivel de confianza esperado & $* 9,488$ \\
Nivel de significancia & 0,027 \\
Gamma & $-0,544$ \\
\hline${ }^{*} p<, 05$. &
\end{tabular}

Los hallazgos muestran que el $\left(X^{2}\right)$ obtenido fue 10,951 en los adultos intermedios y tardíos, superior a 9,488, con un valor de confianza del 0,05, utilizándose 4 grados de libertad. Por otra parte, el valor de significancia 0,004, obtenido en el programa SPSS, es menor de 0,01 o de 0,05. Esto indica que se acepta la hipótesis de investigación, es decir, los sujetos deprimidos difieren de los no deprimidos, atribuyendo a la narrativa prototipo de la depresión un mayor grado de relación con su vida que los sujetos no deprimidos.

La función Gamma se aplicó en razón de que se emplea en tablas de contingencia de cualquier tamaño y su resultado se debe encontrar en el intervalo comprendido entre $-1 \mathrm{y}+1$, siendo -1 una relación negativa perfecta y +1 una relación positiva perfecta.

De igual manera, en la tabla 4 se puede observar el valor hallado del coeficiente gamma, el cual fue de $-0,544$; por lo tanto, se presentó una relación negativa moderada entre los grupos comparados; en consecuencia, a mayor grado de identificación con la narrativa prototipo de la depresión del grupo clasificado como depresivo, menor será el grado de identificación con la narrativa prototipo de la muestra clasificada como no depresiva. Por consiguiente, se puede concluir que las variables están relacionadas en forma significativa.

\section{Discusión}

Teniendo en cuenta la hipótesis de investigación planteada: "Los adultos intermedios y tardíos depresivos de centros de atención al adulto mayor de la ciudad de Bucaramanga, examinados, diferirán de los no depresivos en su atribución de relación de la Narrativa Prototipo de la depresión con su vida". Se pudo observar que los participantes depresivos difieren de los no depresivos. Con esto se comprueba la hipótesis de investigación, es decir, que las personas entrevistadas que no presentaron ninguna patología diferente de la depresión y que alcanzaron un puntaje mayor de 18, en el test de Hamilton, se identificaban en la escala de la narrativa prototipo de la depresión con los grados mayores: tiene relación con mi vida (3), mucha relación con mi vida (4) y total relación con mi vida (5), y que la muestra que no presentaba síntomas de depresión estableció una relación 
con grados menores: ninguna relación con mi vida (1) y alguna relación con mi vida (2). En síntesis, la narrativa prototipo de la depresión tiene un significado especial para el grupo de adultos intermedios y tardíos depresivos que permiten diferenciarlos de la muestra no depresiva.

En cuanto a los objetivos establecidos en este trabajo de investigación se pudo determinar la relación existente entre la identificación de la narrativa prototipo de la depresión de los adultos intermedios y tardíos que presentan síntomas fuertes de depresión y los que no presentan síntomas depresivos; así mismo, se logró verificar la validación convergente de la narrativa prototipo, en un número de sujetos (600) superior al que se ha tenido en cuenta en estudios precedentes.

A pesar de la existencia de diferencias significativas socioculturales entre Colombia y Portugal, se puede observar que en los diversos estudios realizados los sujetos con síntomas depresivos se identificaron de la misma manera con la narrativa prototipo de la depresión con los grados superiores -(3) tiene, (4) mucha y (5) total relación con mi vida)

Sin dejar de lado los antecedentes investigativos, vale la pena tener en cuenta que varios autores han realizado investigaciones relacionadas con la validación de la narrativa prototipo, enfocadas en diversas patologías. Es así como uno de los estudios más importantes en este ámbito es el realizado por Maia (1998) en Portugal, en el cual se encuentra una existencia de la narrativa prototipo en sujetos depresivos, que difieren de los sujetos normales o de aquellos diagnosticados con otras patologías. En estos estudios la muestra utilizada no supera el $20 \%$ con respecto a la empleada en esta investigación, en la cual el número de sujetos participantes fue de 600 , lo que permitió una mayor confiabilidad en los resultados obtenidos.

En esta investigación se pudo observar que los adultos intermedios y tardíos reafirman su discurso patológico, llegando al punto de presentar invariantes prototípicos depresivos, lo cual apoya la idea de la continuidad narrativa prototípica en exploración (Gonçalves y Pocinho, 1989; Gonçal- ves, 1989b). En cuanto a las diferencias socioculturales, se pudo observar que en los estudios realizados en diferentes países los sujetos con síntomas depresivos se identificaron en la misma forma con la escala convergente en la narrativa prototipo de la depresión.

\section{Referencias}

Alves, A. (1993). Organização cognitiva e narrativa prototipo em toxicodependientes. Estudo exploratorio. Tese de maestrado em Psicologia. Universidade do Minho.

Duarte, Z. (1993). Narrativas prototipo e dependencia alcoolica. Tese de maestrado em Psicologia. Universidade do Minho.

Gonçalves, O. (2002). Psicoterapia cognitiva narrativa. Manual de terapia breve. Bilbao: Desclee.

Gonçalves, O., Maia, A., Alves, A.R., Soares, I., Duarte, Z.T. \& Henriques, M. (1986). Narrativas prototipo e psicopatología. Psicologia: teoria, investigação e práctica, 1, 307-318.

Gonçalves, O., \& Machado P.P. (1989). Cognitive therapies and psychological development: An introduction. En O.F. Gonçalves (ed.). Advances in the cognitive therapies, the constructive developmental approach. Porto: Apport, $1-9$.

Gonçalves, O. (1989b). The constructive-developmental trend in cognitive therapies. En: 0. Gonçalves (ed.). Advances in the cognitive therapies: The constructive developmental approach. Porto: Apport.

Gonçalves, O. (1992). Congnitive Narrative Psychotherapy. Comunicação apresentada no III International Conference on Construtivism in Psychotherapy. Barcelona.

Gonçalves, O., Maia, A., Soares, I., Duarte, Z., Alves, A., Henriques, M. \& Pocinho, F. (1992). Prototype narratives, cognitive structures and 
psychopathology: Some preliminary remarks. Comunicação apresentada No World Congress of Cognitive Therapy, Toronto, Canada.

Gonçalves, O. (1995) Auto-conhecimento e acesso introspectivo: do self reificado ao self narrative. Dissertação de doutoramento não publicada. Portugal: Universidade do Minho.

Gonçalves, O., Maia, A., Alves, A.R., Soares, I., Duarte, Z.T. \& Henriques, M. (1996). Narrativas prototipo e psicopatología. Psicología. Teoria, investigação e práctica, 1, 307-318.

Henriques, M. (1995) Narrativas prototipo na agorafobia: estudo exploratorio para a comprensão da construção de significados na agorafobia. Tese do maestrado em Psicologia. Universidade de Coimbra.

Maia, A. (1998). Narrativas prototipo e organização do cohecimiento na depressão. Tese de doutoramento em Psicologia. Universidade do Minho.

Pocinho, F. (1999). Narrativa prototipo e organização do cohecimiento na obsessão-compulsão. Tese de doutoramento em Psicología. Universidade do Minho.

Soares, I. (1995). Narrativa prototipo na anorexia nervosa: estudo exploratorio para a comprensão da organização do cohecimiento na anorexia nervosa. Tese de maestrado em Psicologia, Universidade do Minho.

SCID (Structured interview of mental upheavals based on the DSM-IV).

Test Psicométrico de Depresión de Hamilton ( $\mathrm{Ha}$ milton Depression Scale Rating). 